\title{
Polish Rheumatologists and Rheumatoid Arthritis Patients Differ in Their Opinions About Treatment Inefficacy
}

\author{
Anna RACZKIEWICZ, Aleksandra JUSZKIEWICZ, Malgorzata TLUSTOCHOWICZ, Witold TLUSTOCHOWICZ
}

Department of Internal Diseases and Rheumatology, Military Institute of Medicine, Warsaw, Poland

\begin{abstract}
Objectives: This study aims to detect the differences of opinion between rheumatoid arthritis patients and rheumatologists concerning factors affecting escalation of disease modifying antirheumatic drugs (DMARDs).

Materials and methods: The study included 82 rheumatoid arthritis patients ( 14 males, 68 females; mean age $61.4 \pm 11$ years; range 35 to 84 years) and 85 rheumatologists ( 26 males, 59 females; mean age $49.7 \pm 11.7$ years; range 33 to 77 years). All participants were asked to complete a survey which was composed of nine factors related to the ineffectiveness of RA treatment and the necessity of treatment change or DMARDs escalation. The patients were also asked to assess their satisfaction with current treatment and trust in their doctor. We collected demographic data of patients as well as their Disease Activity Score 28, erythrocyte sedimentation rate, and Health Assessment Questionnaire score.

Results: Of the patients, $83 \%$ declared confidence in their doctor and $74.5 \%$ declared satisfaction with current therapy, while only $44 \%$ achieved low disease activity. The most important reasons for patients to escalate their therapy were persistent pain, functional impairment, progression of erosions, and rheumatologist's decision. For rheumatologists, the most important issues were progression of erosions, joints' swelling, high erythrocyte sedimentation rate, and morning stiffness. Radiological progression, joints' swelling, and high erythrocyte sedimentation rate were considered more important for DMARD escalation by physicians than by patients. Middle-aged rheumatologists of 41-60 years of age and female rheumatologists were more inclined to consider patients' opinion as a factor for treatment change.

Conclusion: Different factors are important for rheumatologists and rheumatoid arthritis patients regarding DMARDs escalation. Treatment satisfaction is determined not only by disease activity indices but also by other patient-oriented factors. Improved communication between patients and physicians plays a significant role in achieving the therapeutic goal of tight control of the disease.

Keywords: Patients; physicians; rheumatoid arthritis; therapy.
\end{abstract}

Rheumatoid arthritis (RA) is a progressive systemic inflammatory disease that requires life-long therapy and effective cooperation between patient and physician. The modern concept of tight control of RA and treating to target consists of early diagnosis, aggressive treatment, and regular monitoring of disease activity to achieve low disease activity or clinical remission. It is increasingly accepted that RA treatment should be based on a shared decision between patient and rheumatologist. ${ }^{1}$ However, not all aspects of arthritis are equally important to different patients, and perceptions of important health status outcomes differ considerably between patients and rheumatologists. The awareness of signs and symptoms of active disease may differ between patients and doctors, and may lead to misunderstanding which may cause delays in seeking medical advice or receiving adjusted therapy. Previous studies that have explored patients' perception of the relative importance of improving different aspects of RA indicate that patients consider pain and physical disabilities to be the most important factors for treatment change. $^{2,3}$ To improve communication and establish real doctor-patient partnership, it is essential to identify the aspects of health status which are perceived differently by patients and 
physicians. Thus, in this study, we aimed to detect the differences of opinion between RA patients and rheumatologists concerning factors affecting escalation of disease modifying antirheumatic drugs (DMARDs).

\section{MATERIALS AND METHODS}

Eighty-two RA patients meeting American College of Rheumatology 1987 criteria (14 males, 68 females, mean age $61.4 \pm 11$ years; range 35 to 84 years) with a mean disease duration of $10.3 \pm 8$ years and treated with DMARDs at the Military Institute of Medicine outpatient clinic between January 01, 2010 - December 31 2010 were included in the study. The inclusion criterion was regular disease monitoring (every 1-3 months) and DMARDs escalation during the last 12 months. All but four patients were treated with methotrexate and the treatment escalation meant escalation of methotrexate dose $(\mathrm{n}=20)$ or adding another DMARD (leflunomide in 56, sulfasalazine in four, and cyclosporine in two patients). The study also included 85 rheumatologists (26 males, 59 females; mean age $49.7 \pm 11.7$ years; range 33 to 77 years) attending the Polish Society for Rheumatology Congress in 2010. All participants were asked to complete a questionnaire consisting nine factors related to the ineffectiveness of RA treatment and the necessity of treatment change or DMARDs escalation. The factors associated with the treatment failure in the questionnaire were persistent joint pain, deterioration of functional ability, persistent swelling of the joints, morning stiffness lasting more than one hour, fatigue, high erythrocyte sedimentation rate (ESR), progression of erosions, rheumatologist's decision about current therapy (patients' questionnaire), patient's opinion about worsening of the disease (rheumatologists' questionnaire), and difficulties in performing paid work. $50 \mathrm{~mm}$ visual analog scale (scored 0-5) was used to illustrate the importance of each factor in an individual opinion. The respondents were encouraged to add other items and comments relevant in their opinions. The patients were also asked to assess their satisfaction with current treatment and trust in their doctor in 5-point Likert's scale. Additionally, we collected data on patients' education, employment, place of living (town with $>100,000$ inhabitants, town with $\leq 100,000$ inhabitants or village), disease activity before treatment escalation and current disease activity (6-12 months after treatment escalation). For this assessment, we used the Disease Activity Score 28 (DAS28) with ESR as a laboratory datum. Functional impairment was assessed using Health Assessment Questionnaire (HAQ). The data for HAQ before and after treatment change were available for 65 patients. In the statistical analysis, we used Statistica version 10.0 (StatSoft). Results are reported as mean \pm standard deviation for continuous variables and $\mathrm{n}(\%)$ for categorical variables. One-way analysis of variance was used for the comparison of independent variables. Spearman correlation coefficient was used to assess the correlation between variables. $\mathrm{P}$ value $<0.05$ was considered statistically significant. Cluster analysis was performed to identify items for which respondents gave similar answers.

\section{RESULTS}

For all participants, the most important factors for treatment change were persistent pain, functional deterioration (both mean 4.47 \pm 0.7 points), radiological progression of joint damage (4.37 \pm 0.4$)$ and rheumatologists' decision about treatment (mean 4.3 \pm 0.4 ) (Figure 1). Twenty-five patients (30.5\%) added drug toxicity or intolerance as an important factor for treatment change. One patient mentioned that even

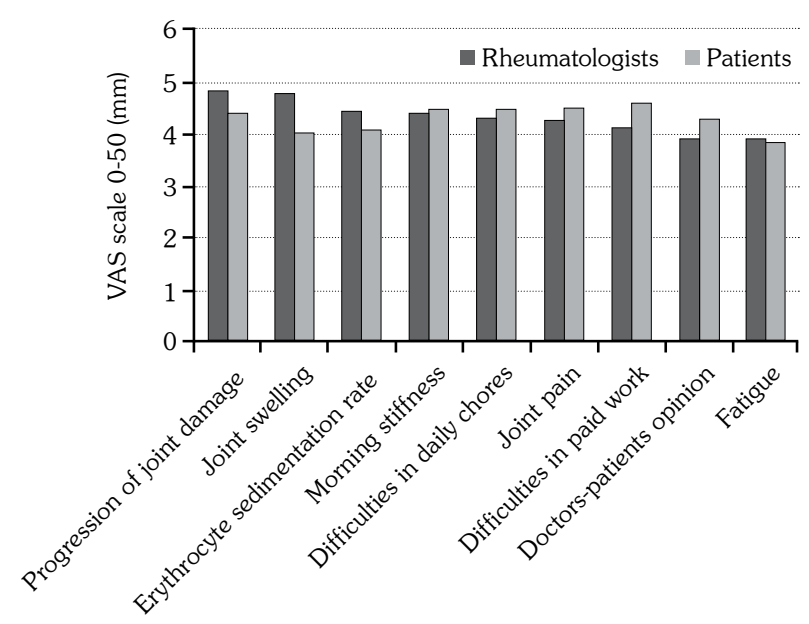

Figure 1. Comparison of rheumatologists' and patients' rating of factors important for treatment escalation. Difficulties in paid work were assessed only by patients $<60$ years old. VAS: Visual analog scale. 
one painful joint was sufficient to decide for treatment escalation. Another patient expressed an opposite opinion saying that since pain is an incurable RA symptom, it should not be taken into consideration regarding disease activity.

Most of the patients lived in cities $(n=49$, 59.7\%), 24 (29.3\%) were from small towns, and nine (11\%) lived in villages. The patients living in smaller towns and villages were slightly more inclined to rely on their doctors' opinion than patients from cities (mean $45 \mathrm{~mm}$ vs. $41 \mathrm{~mm}$ in visual analog scale). Four patients completed eight years of formal education, 10 patients completed 10 years, 32 patients completed $11-12$ years, and 30 patients completed $>12$ years, while these data are missing for six patients. The majority of patients were retired $(n=48,58.5 \%), 18(22 \%)$ were employed, 12 (14.6\%) received pension, and four (4.9\%) were unemployed. Mean DAS28 was $5.34 \pm 1.2$ at the time of treatment escalation and $3.6 \pm 1.3$ at the time of survey. DAS28 remission was achieved by 21 patients (25.6\%) and low disease activity by 15 patients (18.3\%); 35 patients (42.7\%) had moderate disease activity and 11 (13.4\%) still had high disease activity. Mean HAQ (for 65 patients) was $1.34 \pm 0.8$ before and $1.14 \pm 0.8$ after treatment change. There were no significant differences in questionnaire answers in relation to patients' education level, disease duration, sex, functional impairment, and present disease activity. Substantial DAS28 improvement $(>2.5)$ after treatment change was associated with lower rating of deterioration of functional abilities as a reason for treatment escalation $(p=0.044)$. There was a difference in opinion about treatment change in relation to patients' age. The patients older than 60 years assessed the difficulties in performing paid work and persistent swelling of the joints as less important than younger patients did ( $p=0.007$ and $p=0.01$, respectively). The reliance on rheumatologists' decision was more strongly expressed by middle-aged patients (41-60 years old) than by younger and older patients $(p=0.005)$.

Sixty-five patients (14 males and 51 females) assessed their satisfaction with current therapy and trust in their rheumatologists. The majority $(n=54,83 \%)$ declared high or very high confidence in their doctor, nine (13.8\%) medium confidence, and two (3\%) low confidence. High or very high satisfaction with current therapy was expressed by 51 patients (74.5\%), moderate satisfaction by eight (12.3\%), low satisfaction by two (3\%) and dissatisfaction by four (6.15\%) patients. Satisfaction with current treatment correlated moderately and inversely with present HAQ (Figure 2) and DAS28 (correlation coefficient -0.42 and -0.38 , respectively) and to a lesser degree with HAQ and DAS28 improvement after treatment change (correlation coefficient 0.33 and 0.25 , respectively). The level of treatment satisfaction did not differentiate patients' opinion about the causes of treatment change. Higher grading of trust "in your own doctor" was associated with higher rating of fatigue as a variable important for treatment change $(p=0.01)$.

The most important factors for treatment escalation for rheumatologists were radiological progression (mean $4.83 \pm 0.37$ ), swelling of the joints (4.77 \pm 0.5 ), high ESR (mean $4.4 \pm 0.6$ ), and morning stiffness (mean 4.46 \pm 0.7 ) (Figure 1). Eighteen rheumatologists (22\%) added high DAS28 score as an important factor for making a decision about treatment change. Middle-aged rheumatologists (41-60 years old) more strongly considered deterioration of functional abilities and the patients' opinion about the treatment as a reason to DMARD escalation than younger and older rheumatologists $(p=0.027$ and $p=0.03$, respectively). Female rheumatologists were more inclined to take into account their patients' opinion

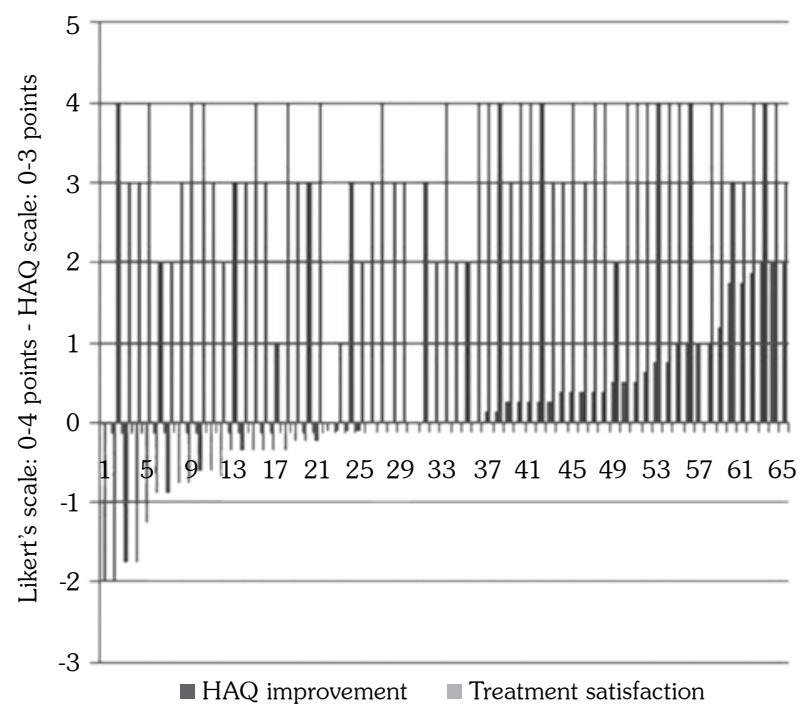

Figure 2. Level of treatment satisfaction (0-4 point Likert's scale) and improvement in Health Assessment Questionnaire (HAQ) in 65 rheumatoid arthritis patients. 
about the treatment than male rheumatologists $(p=0.046)$.

The following factors were considered more important by rheumatologists than by patients: progression of erosions $(p=0.0003)$, persistent swelling of the joints $(p=0.0003)$, high ESR $(p=0.022)$, and difficulties in performing paid work $(p=0.007)$. If only the patients younger than 60 years are taken into account, they were the patients who paid more attention to difficulties in performing paid work than physicians $(p=0.01)$. Persistent joint pain, decline of functional abilities, morning stiffness, and fatigue were equally important for patients and physicians with regard to the aspects of treatment change.

The agglomeration technique has shown that rheumatologists' opinions can be divided into three groups according to the similarity of evaluation scores. In the first group, there were the features related to active inflammation: joint swelling, morning stiffness, high ESR, and erosions. The second group consisted of more subjective issues like persistent pain, deterioration in performing tasks at home and at work, and patients' opinion. The problem of fatigue was seen by rheumatologists as a separate item. There was no such consistency in patients' answers to the questionnaire. The patients evaluated quite similarly: pain, deterioration in performing daily chores, morning stiffness, ESR, worsening of erosions, and fatigue. Separately from those items, they evaluated swelling of the joints, rheumatologist's opinion, and difficulties in performing paid work.

\section{DISCUSSION}

It is widely recognized that physicians often rate patients' health status differently than patients themselves. Regarding RA, physicians have been reported to differ from patients in the assessment of patients' physical and mental function, ${ }^{4}$ disease activity, ${ }^{5}$ and relevance of disability to the patient. ${ }^{6}$ There is limited number of studies concerning physicians/patients concordance in opinion about the need of treatment escalation. In the Quantitative Standard Monitoring of Patients with RA study, based on information obtained from 7,028 patients, pain was the most important determinant of patients' rating of disease activity, followed by fatigue. A higher disease activity rating by patients was associated with older age and a higher proportion of lower educated patients. Rheumatologists' ratings were mostly influenced by the physical examination (swollen and tender joint count) and laboratory markers of inflammation such as ESR. ${ }^{7}$ In our study, the patients' rating was not significantly influenced by the education level and fatigue was less important than other factors assessed both by physicians and patients. However, patients with higher trust "in their doctor" paid more attention to the significance of fatigue. This may reflect the situation in which patients do not tell about all relevant symptoms because they do not have full confidence in their physician.

In a survey composed of 58 factors related to DMARD escalation, the most important reasons to escalate DMARDs for rheumatologists were swollen joint count, DAS28, their global assessment of RA, worsening of erosions, and deterioration of disease activity compared to disease activity three months before. ${ }^{8}$ For patients, the most important issues were the present level of physical functioning, trust in their doctor, number of painful joints, satisfaction with present DMARD, and current general health. ${ }^{8,9}$ We assessed only nine factors, but the results are quite comparable, concerning rheumatologists' opinions about joint swelling and erosions, and the patients' opinions about pain, physical functioning, and physician's decision. In another survey assessing six factors important for treatment escalation (patient's age, change in RA symptoms, change in erosions, DAS28, disease duration, and current treatment), DAS score was the most powerful factor influencing rheumatologists' decision. ${ }^{10} \mathrm{We}$ did not include DAS28 in our questionnaire because the patients were not familiar with DAS28 and we wanted to have doctors and patients fill in the same form. Nevertheless, 22\% rheumatologists added DAS28 as an important factor for their decision process. Apparently, this is an expression of successful endorsement of the standardized RA treatment, which should be based on objective disease activity indicators. Unfortunately, disease activity is a medical term, not always clearly understood by patients. For patients, more understandable treatment goals are relief of physical symptoms and achieving optimal physical and social functioning. ${ }^{11,12}$ 
Our study shows that joint swelling without pain or progression of erosions sometimes is not linked to disease activity by patients. Particularly, older patients may not perceive abnormal joints or be unaware of the importance of the presence of swollen joints in the assessment of RA activity. It has been shown that elderly RA patients with an equal prevalence of comorbidities receive less aggressive treatment than younger RA patients. ${ }^{13}$ The cause may be partially related to patients' unawareness of important symptoms.

In our study, $30.5 \%$ of patients considered drug intolerance as a reason for treatment change - the factor not mentioned by the rheumatologists. Some studies exploring patients' beliefs about DMARDs have revealed anxieties about using these agents, which were perceived as powerful and toxic. DMARDs were accepted by patients as essential to RA management, but strong concerns were expressed about potential long-term effects, even about shortening of life. The patients were inclined to limit the DMARDs dose to the minimum necessary and were not always willing to accept life-long therapy. ${ }^{14-16}$ Additionally, we found a troublesome discrepancy between a widely declared treatment satisfaction (74.5\%) and quite a small number of patients with low disease activity or remission (44\%). This finding is concordant with the results of a large study comprising over 6,000 patients, where more than three-fourths of the patients were satisfied with their therapy, but the majority of those patients had moderate or even high arthritis activity. ${ }^{17}$ Satisfaction with RA control and risk of side effects were the dominant cause of unwillingness to change the therapy. ${ }^{17}$ In another study, 65\% of 97 patients with moderate to high disease activity did not want to change their current medications. Major causes of unwillingness to change were satisfaction with current disease state and perceived risk of side effects. ${ }^{18}$ These data indicate that there is an important discrepancy between declared satisfaction with therapy and measured activity and functional status, and that clinical activity is not an adequate explanation for treatment satisfaction. Health professionals should take into account possible fear for drug toxicity as a cause of unwillingness to treatment change or noncompliance; otherwise, we may be in a disappointing situation in which patient and doctor are satisfied, but the disease is not controlled adequately. A recent study concerning patients' expectation of RA treatment has shown that the patients, who had experienced clinical remission, increased their expectations of treatment and hoped to have their symptoms not alleviated but eradicated. ${ }^{19}$ It is possible that the majority of our "satisfied" patients did not experience remission, so they did not believe the disease was manageable. In a large community-based study concerning the problem of risk perception and unwillingness to try another DMARD by RA patients, the patients "resistant to change" were significantly older, used fewer DMARDs before, had lower health literacy as well as higher perception of medication risk. ${ }^{20}$ Low income, depression, and years of education were not significantly related to their willingness to take proposed DMARD. ${ }^{20}$ The clinical implication is that lower health literacy, as an indicator of cognitive problems, regardless of the level of education, may be one of the risk factors for patient/physician discordance.

Our study mostly confirms earlier findings, but it also reports some new findings. Not only the patient's, but also rheumatologist's age seems to be important regarding approach to treatment change. In our study, middle-aged rheumatologists more often than older and younger physicians declared intention to incorporate patients' opinion in the decision about further treatment. Unfortunately, we did not ask rheumatologists about the scope of their current medical practice and the years of rheumatologic experience. Supposedly not the age makes the difference, but the years of clinical experience and the intensity of current professional activity.

Our study has several limitations: we recruited our patients from only one center, all the patients were on conventional DMARDs therapy, and we investigated declarations about the decision process, not the real process. In addition, the study was performed four years ago, before the wide endorsement of "Treat to target" recommendations. Despite these limitations, we believe our results shed some light into outcomes important to Polish patients and doctors, which might contribute to treatment decisions. While starting a new DMARD, clinicians should be aware of the possibility of cognitive impairment or age related decline in information processing that could affect the patient's decision making. Considering patients' perspectives and gaining 
patient's cooperation may help to achieve better treatment outcomes.

In conclusion, different factors are important for rheumatologists and RA patients regarding treatment failure and necessity of DMARDs escalation. Treatment satisfaction is determined not only by disease activity indices but also by other patient-oriented factors. Improved communication between patients and physicians is important in achieving the therapeutic goal of tight control of the disease.

\section{Declaration of conflicting interests}

The authors declared no conflicts of interest with respect to the authorship and/or publication of this article.

\section{Funding}

The authors received no financial support for the research and/or authorship of this article.

\section{REFERENCES}

1. Smolen JS, Landewé R, Breedveld FC, Buch M, Burmester G, Dougados M, et al. EULAR recommendations for the management of rheumatoid arthritis with synthetic and biological diseasemodifying antirheumatic drugs: 2013 update. Ann Rheum Dis 2014;73:492-509.

2. Heiberg T, Kvien TK. Preferences for improved health examined in 1,024 patients with rheumatoid arthritis: pain has highest priority. Arthritis Rheum 2002;47:391-7.

3. Kwoh CK, Ibrahim SA. Rheumatology patient and physician concordance with respect to important health and symptom status outcomes. Arthritis Rheum 2001;45:372-7.

4. Suarez-Almazor ME, Conner-Spady B, Kendall CJ, Russell AS, Skeith K. Lack of congruence in the ratings of patients' health status by patients and their physicians. Med Decis Making 2001;21:113-21.

5. Cadart S, Kanagaratnam L, Corli L, Flipo R-M. Doctor/patient math in disease evaluation activity in rheumatoid arthritis. Ann Rheum Dis 2013;72:A897.

6. Hewlett S, Smith AP, Kirwan JR. Values for function in rheumatoid arthritis: patients, professionals, and public. Ann Rheum Dis 2001;60:928-33.

7. Khan NA, Spencer HJ, Abda E, Aggarwal A, Alten $\mathrm{R}$, Ancuta $\mathrm{C}$, et al. Determinants of discordance in patients' and physicians' rating of rheumatoid arthritis disease activity. Arthritis Care Res (Hoboken) 2012;64:206-14.
8. van Hulst $\mathrm{L}$, Wietske $\mathrm{K}$, van Riel P, Fraenkel $\mathrm{L}$. Different Factors Are Important for RA Patients and Rheumatologists Regarding DMARD Escalation. Arthritis Rheum 2010;62 Suppl 10:59. [Abstract]

9. van Hulst LT, Kievit W, van Bommel R, van Riel PL, Fraenkel L. Rheumatoid arthritis patients and rheumatologists approach the decision to escalate care differently: results of a maximum difference scaling experiment. Arthritis Care Res (Hoboken) 2011;63:1407-14.

10. Kievit W, van Hulst L, van Riel P, Fraenkel L. Factors that influence rheumatologists' decisions to escalate care in rheumatoid arthritis: results from a choicebased conjoint analysis. Arthritis Care Res (Hoboken) 2010;62:842-7.

11. Van der Elst K, Meyfroidt S, De Groef A, Binnard E, Moons $\mathrm{P}$, Verschueren $\mathrm{P}$, et al. Outcomes important to patients with early rheumatoid arthritis. A qualitative interview study. Ann Rheum Dis 2013;72:A358-9.

12. Sanderson T, Morris M, Calnan M, Richards P, Hewlett S. What outcomes from pharmacologic treatments are important to people with rheumatoid arthritis? Creating the basis of a patient core set. Arthritis Care Res (Hoboken) 2010;62:640-6.

13. Radovits BJ, Fransen J, Eijsbouts A, van Riel PL, Laan RF. Missed opportunities in the treatment of elderly patients with rheumatoid arthritis. Rheumatology (Oxford) 2009;48:906-10.

14. Fraenkel L, Bogardus S, Concato J, Felson D. Unwillingness of rheumatoid arthritis patients to risk adverse effects. Rheumatology (Oxford) 2002;41:253-61.

15. Goodacre LJ, Goodacre JA. Factors influencing the beliefs of patients with rheumatoid arthritis regarding disease-modifying medication. Rheumatology (Oxford) 2004;43:583-6.

16. Kumar K, Gordon C, Barry R, Shaw K, Horne R, Raza K. 'It's like taking poison to kill poison but I have to get better": a qualitative study of beliefs about medicines in Rheumatoid arthritis and Systemic lupus erythematosus patients of South Asian origin. Lupus 2011;20:837-44.

17. Wolfe F, Michaud K. Resistance of rheumatoid arthritis patients to changing therapy: discordance between disease activity and patients' treatment choices. Arthritis Rheum 2007;56:2135-42.

18. Takahashi N, Sasaki K, Nishiyama T, Naniwa T. Satisfaction and attitudes toward therapy in patients with rheumatoid arthritis. Mod Rheumatol 2012;22:376-81.

19. Funahashi K, Matsubara T. What RA patients expect of their treatment--discussion over the result of our survey. Clin Rheumatol 2012;31:1559-66.

20. Martin RW, McCallops K, Head AJ, Eggebeen AT, Birmingham JD, Tellinghuisen DJ. Influence of patient characteristics on perceived risks and willingness to take a proposed anti-rheumatic drug. BMC Med Inform Decis Mak 2013;13:89. 